\title{
Erratum: Unraveling the composition dependence of the martensitic transformation temperature: A first-principles study of Ti-Ta alloys [Phys. Rev. B 94, 224104 (2016)]
}

\author{
Tanmoy Chakraborty, Jutta Rogal, and Ralf Drautz
}

(Received 7 June 2017; published 22 June 2017)

DOI: 10.1103/PhysRevB.95.219905

In the original paper we state in Sec. III that the atomic positions of the $\beta$ phase (austenite) were fully relaxed. This does, however, only apply to the calculations for the $\alpha^{\prime \prime}$ phase (martensite), whereas for the $\beta$ phase atomic positions were kept at ideal bcc positions, and only the volume of the supercells was optimized.

Since at $T=0 \mathrm{~K}$ the $\beta$ phase in Ti-Ta alloys is unstable in the investigated composition range, the structures directly relax into the stable $\alpha^{\prime \prime}$ phase upon full optimization of all degrees of freedom. We therefore chose a constrained approach with fixed atomic positions for the $\beta$ phase.

We would like to thank A. Ferrari for pointing out the discrepancy between the energies of the $\beta$ phase for fully relaxed structures that already closely resemble the $\alpha^{\prime \prime}$ phase and structures with optimized volume and fixed atomic positions as reported in our article. 